\title{
Microorganisms Associated with Hydrogel Contact Lens Wear
}

\author{
R.B. Simmons, D.G Ahearn. \\ Biology Department, Georgia State University, Atlanta GA 30303 USA
}

Infectious keratitis with a potential to cause irreversible blindness is a rare but grave complication associated with contact lens wear. The majority of the keratitis cases are caused by bacteria (an incidence of 1-4 per 10,000 contact lens wearers per year) that respond successfully and readily to therapy, but some cases, particularly by the Gramnegative bacterium Pseudomonas aeruginosa and at lesser incidences fungi of the Fusarium solani-F. oxysporum species complexes and amoeba of the genus Acanthamoeba are increasingly difficult to treat. Typically fungal keratitis can be linked to ocular trauma, immunosuppression, or some form of chronic ocular surface illness. On rare occasions keratitis outbreaks have been related directly to specific materials or regimens. Beginning in March of 2005 and extending through the first four months of 2006 an outbreak of fungal keratitis was recorded, first in Singapore and then spreading world wide [1,2]. The outbreak was associated with the use of a single lens care solution. The solution ( $\mathrm{ReNu}$ with MoistureLoc) was subsequently removed from the market by the manufacturer. Solutions as commercially available were not contaminated with Fusarium, but they were subject to contamination during the stresses of consumer use and noncompliance with good hygienic practice. In 2007 concern about an apparent increase in lens wear associated Acanthamoeba keratitis resulted in the voluntary recall of another lens care product [3]. Again this was an issue of apparent product incapacity rather than manufacturer contamination.

Microbial contamination of contact lenses, contact lens care solutions (particularly in the lens storage case) and the lens cases themselves is of concern for manufacturers as well as consumers. Survival of potentially pathogenic organisms in these products and subsequent inoculation into the eye can lead to infection. Organisms that readily adhere to hydrogel lenses and fungi that may penetrate the matrices of hydrogel lens may be associated with increased risks of infection $[4,5]$. Fungi and many protozoa such as Acanthamoeba produce asexual stress-resistant resting stages (chlamydoconidia and cysts respectively) which provide for their survival in many antimicrobial products. Induction of chlamydoconidia and cyst formation in lens care products is of particular concern due to the recalcitrant nature of ophthalmic infections by these organisms. Infections by eukaryotic organisms present particular difficulties in drug therapy due to similarities in physiology with host cells.

Microscopy has played an important role in both the research and clinical areas of microbial keratitis studies. The presence of and possible colonization by microorganisms associated with lenses, lens care solutions, solution containers and lens storage cases may often be demonstrated by the application of imaging technologies to studies of these materials. Application of microscopic imagery to the evaluation of lens care solutions' activity against protozoan microorganisms such as Acanthamoeba appears particularly promising for practical demonstrations of efficacy. 
[1] D.C. Chang, et al. JAMA 296 (2006) 953-963.

[2] W-B Khor, et al. JAMA 295 (2006) 2867-2873.

[3] Food and Drug Administration 2007 http://www.fda.gov/oc/po/firmrecalls/amo05_07.html

[4] L.A. Wilson and D.G. Ahearn. Am. J. Ophthalmol. 101 (1986) 434.

[5] R.B Simmons, et al. J. Clin.Microbiol. 24 (1986) 21-25.

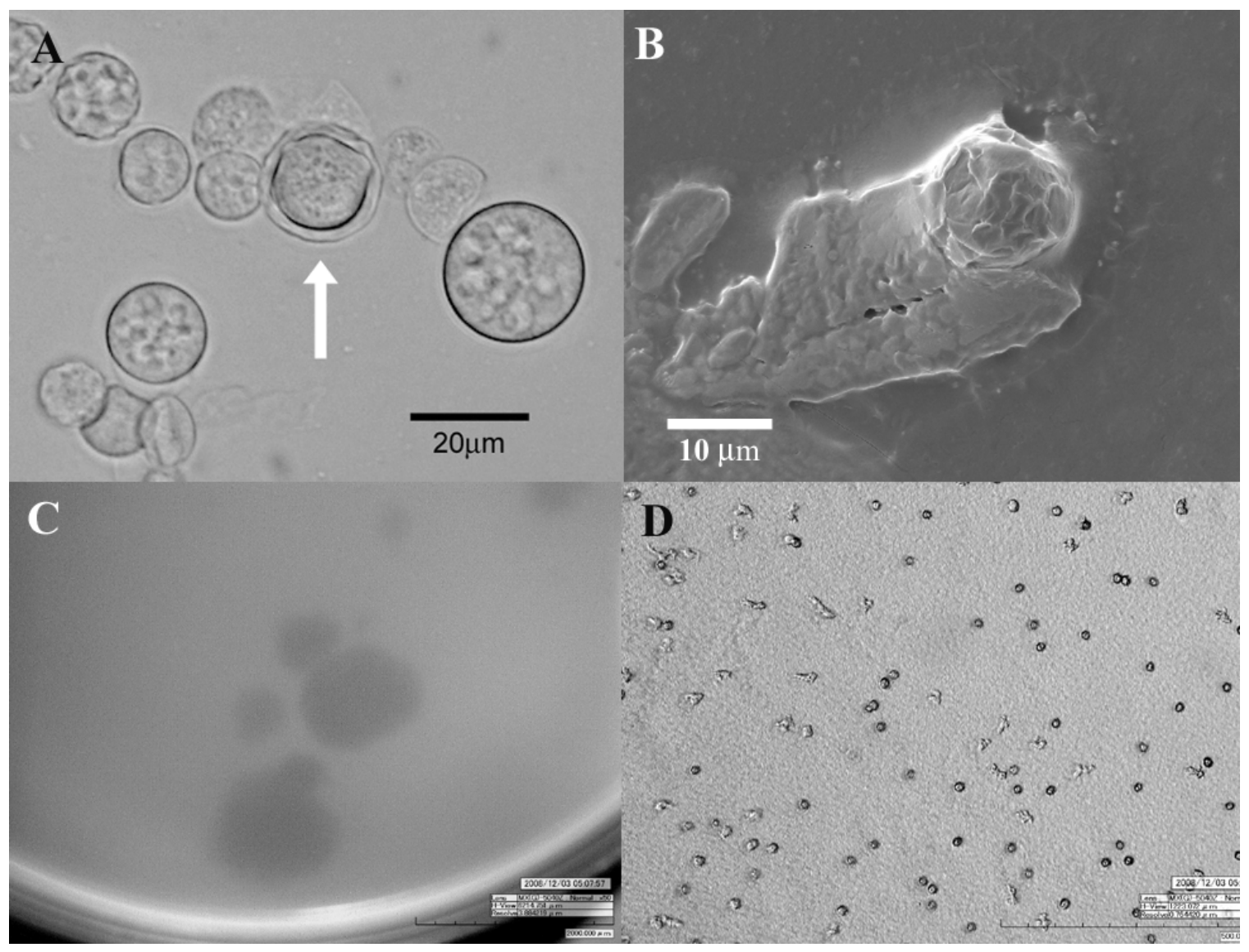

Figure 1. Acanthamoeba castellanii associated with contact lens care materials. A. Cyst formation showing immature and mature cysts (arrow). B. Mature cyst in a dried lens disinfection solution film. C. Plaque assay used in assessment of disinfection solution efficacy. D. Light micrograph showing multiple cysts and trophozoites present in a single plaque. 